\title{
Ligand Binding: Molecular Mechanics Calculation of the Streptavidin-Biotin Rupture Force
}

\author{
Helmut Grubmüller, ${ }^{*}$ Berthold Heymann, Paul Tavan
}

The force required to rupture the streptavidin-biotin complex was calculated here by computer simulations. The computed force agrees well with that obtained by recent single molecule atomic force microscope experiments. These simulations suggest a detailed multiple-pathway rupture mechanism involving five major unbinding steps. Binding forces and specificity are attributed to a hydrogen bond network between the biotin ligand and residues within the binding pocket of streptavidin. During rupture, additional water bridges substantially enhance the stability of the complex and even dominate the binding interactions. In contrast, steric restraints do not appear to contribute to the binding forces, although conformational motions were observed.

Molecular recognition is a prerequisite for information processing in biological systems and is realized by specific ligand-receptor interactions. Despite progress in obtaining experimental data on such interactions, there is little known about the binding and unbinding reaction pathways and about the molecular basis for the specificity of the reactions. This lack of knowledge arises from the difficulty in bridging apparent gaps between experimental data obtained by different techniques. On the one hand, x-ray or nuclear magnetic resonance (NMR) studies provide structural information at atomic resolution, but typically yield static pictures-for example, of bound and unbound states. On the other hand, experiments that probe the binding kinetics rarely pertain to atomic details.

Recent atomic force microscope (AFM) experiments (Fig. 1A) have probed the force required to rupture single streptavidin-biotin complexes $(1,2)$ and have provided additional insights into the binding properties of this well-known model system (3). By measuring binding forces, these AFM experiments have provided a new view of ligand-receptor interactions (1). Conventionally, experiments on ligand binding deal with binding free energies; these have been calculated for the streptavidin-biotin complex by molecular dynamics (MD) simulations in combination with free energy perturbation techniques (4).

However, from such calculations one cannot derive the rupture force measured in the AFM experiments for the following reason. The perturbation techniques mentioned above, on the one hand, use a nonphysical reaction pathway to compute the free energy difference between bound and unbound states. The rupture force, on the other hand, is the largest force along the

Theoretische Biophysik, Institut für Medizinische Optik, Ludwig-Maximilians-Universität München, Theresienstraße 37, D-80333 München, Germany.

*To whom correspondence should be addressed. E-mail: Helmut.Grubmueller@Physik.uni-muenchen.de actual unbinding reaction pathway and is given by the steepest slope in the free energy profile along that pathway (5). It is, therefore, a function of the shape of the free energy profile and generally has no relation to the value of the binding free energy. The AFM data show that this is true for the streptavidin-biotin complex (1).

To reveal the microscopic processes underlying the AFM observations, we present here a theoretical approach using extended MD simulations (6) of a large protein-solvent system. Because of recent algorithmic and technological advances (7), we were able to avoid nonphysical truncations of long-range forces, which up to now have been inevitable in simulations of that size. Our nonequilibrium approach might appear both obvious and unusual. In our computer simulations (Fig. 1B), we simply pulled the biotin out of the streptavidin binding pocket and measured the required force-that is, we tried to model the AFM experiment as closely as possible (8).

As a starting point for our simulations,

we used the $x$-ray coordinates (9). To reduce the computational cost, we simulated only a streptavidin monomer; we do not expect this restriction to affect our results. All MD simulations were carried out in water solvent (10). As indicated by the symbolic "spring" in Fig. 1B, we pulled the biotin out of the binding pocket in the $z$ direction by subjecting the oxygen atom O2, which in the AFM experiment was connected to the cantilever through a linker molecule, to a harmonic potential

$$
V_{\text {spring }}=k_{0}\left[z_{\mathrm{O} 2}(t)-z_{\text {cant }}(t)\right]^{2} / 2
$$

acting on the $z$ coordinate of atom $\mathrm{O} 2$ $\left(z_{\mathrm{O} 2}\right)$. Here, $k_{0}$ is the spring constant and $z_{\text {cant }}(t)$ is the cantilever position. This spring potential, centered at

$$
z_{\text {cant }}(t)=z_{\text {cant }}(0)+v_{\text {cant }} t
$$

serves to model the elastic cantilever; accordingly, $V_{\text {spring }}$ was shifted toward the right (positive $z$ direction) with cantilever velocity $v_{\text {cant }}$ during each simulation, while the protein's center of mass was kept in place such that the protein was free to adjust to the pulling force by rotational and internal motions. Each simulation was started with $z_{\text {cant }}(0)=z_{\mathrm{O} 2}(0)$, such that at $t=$ 0 , the spring was relaxed. The spring constant $k_{0}$ was chosen as $2.8 \mathrm{~N} \mathrm{~m}^{-1}$ (11). As in the AFM experiment, the binding force was measured by use of Hooke's law by observing the deflection $z_{\mathrm{O} 2}(t)-z_{\text {cant }}(t)$ of the cantilever as a function of cantilever position $z_{\text {cant }}(t)$. As in the AFM experiment, our simulated rupture force was defined as the largest force observed during the simulated unbinding process.

The only major difference between the AFM experiment and our simulation concerns the value for the pulling velocity $v_{\text {cant }}$. Whereas the AFM experiment was carried

Fig. 1. Experimental setups. (A) AFM rupture experiment (1). Biotin molecules (black ball models) were fixed through linker molecules to the cantilever tip (right) as well as to an agarose bead (left). Additionally, the biotin molecules at the cantilever were complexed with streptavidin tetramers (only the backbone is shown), whereas most of the biotin molecules at the bead were blocked with soluble streptavidin. The cantilever was brought into contact with the agarose bead, and typically only a few streptavidin-biotin complexes were formed. As the cantilever was subsequently retracted, the biotin molecules were pulled out of the streptavidin binding pocket, more or less one after the other. Eventually, one single complex remained for a short period of time. In this case, the binding force of this single ligand-receptor pair at the point of rupture could be measured by observation of the deflection of the cantilever. (B) Computer simulation. One biotin molecule and one streptavidin monomer (shown as a ribbon model) were considered. The biotin was pulled out of the binding pocket through a harmonic potential (the "spring"), which was moved with constant velocity $v_{\text {cant }}$ (arrow), while the streptavidin was kept in place. The pulling force acted on the same biotin atom (O2) to which the linker is connected in the AFM experiment (23). 
out on a millisecond time scale, our simulations were limited to nanoseconds; therefore, we had to consider thermal fluctuations and dissipation. Because of these nonequilibrium phenomena, the rupture force should vary systematically with rupture speed (2), and thus the computed rupture forces should be able to be extrapolated to the experimental time scale. To do this, we carried out a series of simulations with pulling velocities ranging from $0.4 \AA \mathrm{ps}^{-1}$ down to $0.015 \AA \mathrm{ps}^{-1}$. The apparent linear dependency of the computed rupture force on pulling velocity at velocities less than 0.15 $\AA \mathrm{ps}^{-1}$ (Fig. 2) suggests that simple friction, described by a friction coefficient of $20 \mathrm{pN}$ $\mathrm{s} \mathrm{m}^{-1}$, dominates the nonequilibrium effects in this regime (12). At velocities greater than $0.2 \AA \mathrm{ps}^{-1}$, the increase of rupture force saturates.

In the AFM experiment, individual rupture forces scatter considerably with a standard deviation of about $50 \mathrm{pN}$ (1). Of course, that scatter could be attributed exclusively to experimental error. However, the computed rupture forces also scattered considerably (Fig. 2), with a standard deviation from the linear fit (for values of $v_{\text {cant }}$ less than $0.15 \AA \mathrm{ps}^{-1}$ ) of roughly $35 \mathrm{pN}$-nearly as large as the observed value of $50 \mathrm{pN}$. That scatter of computational results is due to a heterogeneity of reaction pathways observed in our simulations and is related to the known structural microheterogeneity of proteins commonly described in terms of conformational substates (13).

The good agreement between the results obtained in the AFM experiment and those obtained by our simulations enables us to suggest a detailed rupture mechanism, which we base on the simulations with slow-pulling velocities and, in particular, the slowest one with $v_{\text {cant }}=0.015 \AA \mathrm{ps}^{-1}$

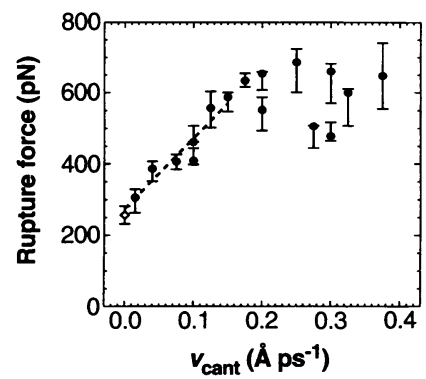

Fig. 2. Computed rupture forces (closed circles) and the experimental rupture force (open diamond) as a function of pulling velocity $v_{\text {cant }}$. For some of the velocities, two computer simulations with slightly different initial conditions were performed. For the computed forces, the error bars give an estimated uncertainty (24). The dashed line shows a linear fit to the computed forces for values of $v_{\text {cant }}$ less than $0.15 \AA \mathrm{ps}^{-1}$. Note that the experimental pulling velocity is close to 0 at the chosen scale.
(14). In the force profile obtained from this simulation (Fig. 3A), the apparent multitude of force maxima mirrors the complexity of the energy landscape traversed by the biotin on its way out of the binding pocket. Here, the force peaks may be attributed to the rupture of short-range interactions like those of hydrogen bonds. This view is supported by the observation that the rupture of several dominant hydrogen bonds and water bridges correlates well with force maxima.

We obtained further insight by inspecting the motion of biotin during rupture (Fig. 3B). The ureido ring moved in a series of steps, each of which was preceded by a force peak. The sudden displacement of the ring at $z_{\text {cant }}=5 \AA$ was caused by rupture of apparently strong hydrogen bonds, whose preceding elongation gave rise to the largest force peak shown in Fig. 3A. By identifying this event as the experimental point of rupture, we obtained an effective rupture length of $5 \AA$, which is within the range of values estimated from the AFM experiments (1). A second force maximum appeared at $z_{\text {cant }}=9 \AA$. It was smaller than the first and has not been observed by AFM, as only the maximum force has been able to be measured.

To explain how the measured forces

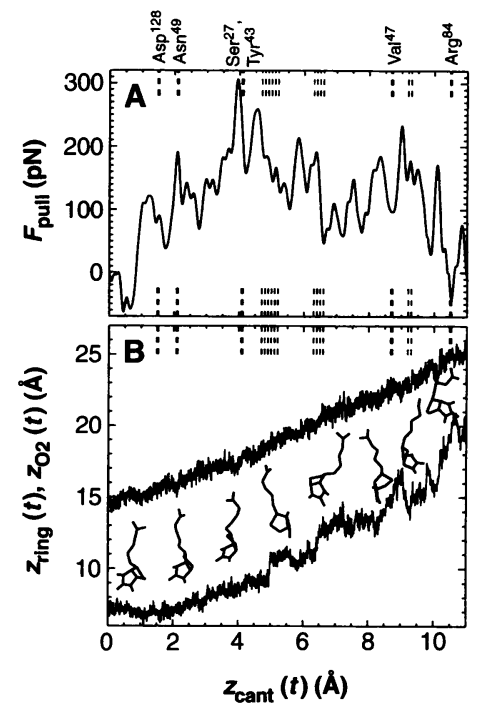

Fig. 3. (A) Pulling force exerted on the biotin molecule as a function of cantilever position $z_{\text {cant }}(t)$ at a pulling velocity of $0.015 \AA \mathrm{ps}^{-1}$ (24). The dashed vertical lines mark the ruptures of hydrogen bonds (bold lines) and water bridges (thin lines) between the biotin and the indicated residues of the streptavidin binding pocket. (B) "Snapshot" of the motion of the biotin. The position of the oxygen atom $\mathrm{O} 2$ (see Fig. 1B) (upper curve), on which the pulling force was exerted, and the position of the center of mass of the ureido ring (lower curve), which points toward the interior of the binding pocket, are plotted as a function of cantilever position $z_{\text {cant }}(t)$. Eight "snapshots" of the biotin structure during rupture are depicted. arise from local interactions between biotin and the streptavidin binding pocket, we took a series of five "snapshots" that characterize major steps of the rupture process (Fig. 4). The bound complex (Fig. 4A) is stabilized by a network of hydrogen bonds (15) and a large number of water bridges between the polar ureido ring " $R$ " of the biotin and selected residues of the binding pocket. The strongest hydrogen bond (approximately $6 \mathrm{kcal} \mathrm{mol}^{-1}$ ) is formed to $\mathrm{Asp}^{128}$; the combination of the two bonds to $\mathrm{Ser}^{27}$ and $\mathrm{Tyr}^{43}$ is of similar strength. Additionally, the oxygen atom $\mathrm{O} 2$ is weakly hydrogen bonded to Asn ${ }^{49}$.

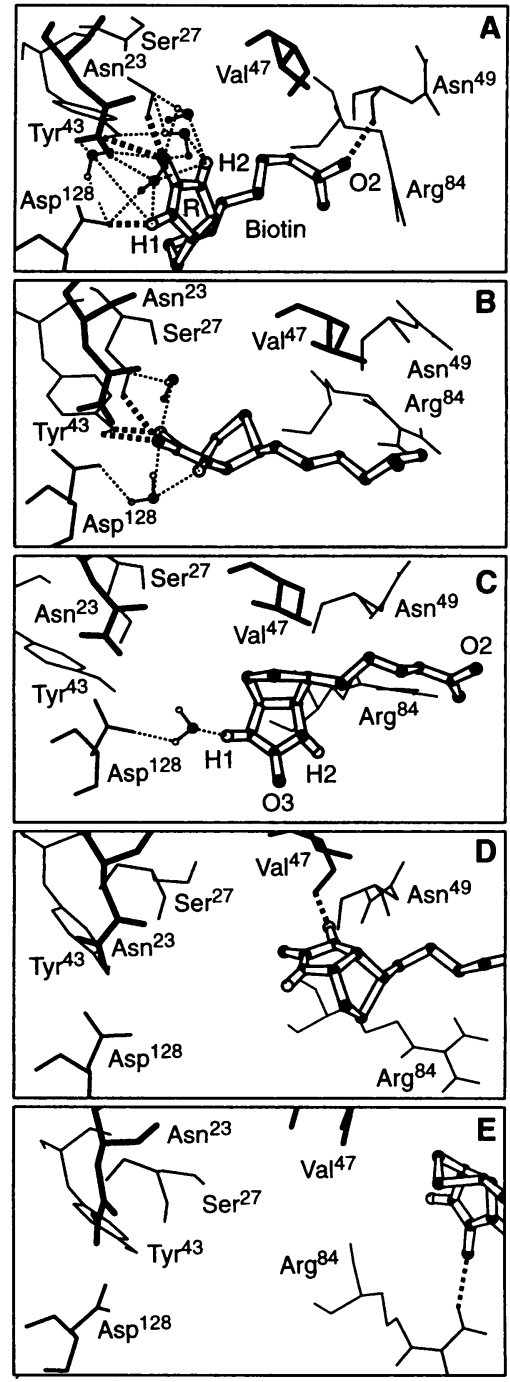

Fig. 4. "Snapshots" of rupture. Biotin is drawn here as a ball-and-stick model (hydrogen atoms, white; heavy atoms, black) within the streptavidin binding pocket; the dashed lines show hydrogen bonds (bold) and water bridges (thin), from which only a selection is shown. The relevant residues of the binding pocket are drawn with depth-cued lines; only polar hydrogen atoms are shown. "Snapshots" were taken (A) at the start of the simulation $\left(t=0, z_{\text {cant }}=0\right)$ and after (B) $200 \mathrm{ps}$ $\left(z_{\text {cant }}=3.0 \AA\right)$, (C) 340 ps $\left(z_{\text {cant }}=5.1 \AA\right)$, (D) 520 ps $\left(z_{\text {cant }}=7.8 \AA\right)$, and $(\mathbf{E}) 710$ ps $\left(z_{\text {cant }}=10.65 \AA\right)$, respectively (23). 
After the cantilever was moved by $z_{\text {can }}$ $=1.5 \AA$, the hydrogen bond to Asp ${ }^{128}$ ruptured, as did subsequently the weak bond to $\mathrm{Asn}^{49}$ (compare the dashed lines in Fig. 3). We were surprised to see, however, that despite a pulling force of more than $100 \mathrm{pN}$, this destabilization did not seem to provoke considerable unbinding motions of the biotin (Fig. 4B; see also the second "snapshot" in Fig. 3B). Rather, the particular architecture of the pocket allowed those water molecules, which are involved in the network of water bridges, to rearrange such as to keep the ureido ring almost in place (for the sake of clarity, only two of the many water molecules are plotted in Fig. 4B). A force larger than $250 \mathrm{pN}$ is required to rupture this network and the two remaining hydrogen bonds. Only after such a force was attained was the biotin released and actually "flicked" forward by $5 \AA$ in two steps (Fig. 4, C and D). This motion was accompanied by a reorientation of the ring and by structural rearrangements within the outer part of the binding pocket, as exemplified by the considerable motion of $\mathrm{Arg}^{84}$ (Fig. 4, A through D). However, because substantial conformational motion occurs only after the force maximum, the induced rearrangements do not seem to contribute much to the rupture force.

At this stage, the polar ureido ring approached $\mathrm{Val}^{47}$ and, after further rearrangement, formed a hydrogen bond to this residue (Fig. 4D; additional water bridges are not shown). This hydrogen bond, in combination with a subsequently established bond to $\mathrm{Arg}^{84}$ (Fig. 4E), gave rise to the second major force barrier. The rupture process was completed only after the latter hydrogen bond broke, which caused further deformation of the binding pocket entry. During the whole rupture process, the biotin molecule covered a distance as large as $12 \AA$. This total rupture length is considerably larger than the effective rupture length in the AFM experiment, as the latter does not include the second force barrier.

Our simulations here provide detailed insight into the complex mechanisms of streptavidin-biotin rupture. They attribute the binding force to a network of hydrogen bonds between the ligand and the binding pocket. In particular, the simulations show that water bridges actively and substantially enhance the stability of the complex. Further work is required to also understand the energetics of the rupture process. Upon extending our approach, it should be possible to quantify the suggested enthalpic nature (16) of the streptavidin-biotin rupture. Similar studies should allow the prediction of the effects of point mutations or ligand substitutions on binding forces for various systems.
Note added in proof: A calculation of the rupture force for iminobiotin-streptavidin yields $125 \pm 20 \mathrm{pN}$ in comparison to the atomic force microscopy measurement of $135 \pm 15 \mathrm{pN}(1)$. A preliminary examination of the rupture process indicates that the unbinding pathway differs from that for the biotin-streptavidin system.

\section{REFERENCES AND NOTES}

1. E.-L. Florin, V. T. Moy, H. E. Gaub, Science 264, 415 (1994); V. T. Moy, E.-L. Florin, H. E. Gaub, ibid. 266, 257 (1994).

2. G. U. Lee, D. A. Kidwell, R. J. Colton, Langmuir 10, 354 (1994).

3. N. M. Green, Adv. Protein Chem. 29, 85 (1975); O. Livnah, E. A. Bayer, M. Wilchek, J. L. Sussman, Proc. Natl. Acad. Sci. U.S.A. 90, 5076 (1993).

4. S. Miyamoto and P. A. Kollman, Proteins 16, 226 (1993)

5. Reviewed in R. Elber, in New Developments in Theoretical Studies of Proteins, R. Elber, Ed. (World Scientific, Tai Seng, Singapore, in press)

6. J. A. McCammon, B. R. Gelin, M. Karplus, Nature 267, 585 (1977); W. F. van Gunsteren and H. J. C Berendsen, Mol. Phys. 34, 1311 (1977)

7. For algorithmic advances, see $C$. Niedermeier and $P$. Tavan, J. Chem. Phys. 101, 734 (1994); M. Eichinger, H. Grubmüller, H. Heller, P. Tavan, in preparation; and H. Grubmüller, Phys. Rev. E 52, 2893 (1995) and references therein. For parallel computing, see (17) and H. Heller, H. Grubmüller, K. Schulten, Mol. Simul. 5, 133 (1990).

8. Alternatively, one may compute the free energy profile along the unbinding reaction path from the MD simulation and derive the rupture force from that profile. However, because free energies pertain to systems in equilibrium, this suggested equilibrium approach rests on the questionable assumption that the rupture is a quasi-stationary process. Furthermore, it requires computations of free energies that notoriously exhibit slow convergence. Thus, in this case our nonequilibrium approach appears to be more appropriate and is computationally less expensive.

9. P. C. Weber, D. H. Ohlendorf, J. J. Wendoloski, F. R Salemme, Science 243, 85 (1989). The X-ray structure contains only residues 13 through 133 of the total of 159 residues. However, this cleavage does not considerably affect the binding affinity. The structure suggests that the interaction between the loop containing $\operatorname{Trp}^{120}$ of the neighboring streptavidin monomer and the biotin in the binding pocket is considerably weaker than that for avidin (3).

10. The simulations were performed with the program EGO (17), which uses the CHARMM force field (18) New multiscale approximations (7) enabled us to consider the full Coulomb interaction (with a dielectric constant equal to 1)-that is, no cutoff (18) was applied. For biotin, the partial charges were taken from (4), and the force constants from (19). All simulations were carried out with an integration step size of $1 \mathrm{fs}$. The lengths of chemical bonds involving hydrogen atoms were fixed (6), nonpolar hydrogen atoms were described through compound atoms (18), and no explicit term for the hydrogen bond energy was included. To quantify the strength of hydrogen bonds, we used a combination of energetic (mainly electrostatics) and steric criteria, as suggested in (20). The protein-ligand complex was placed within a sphere of TIP3 (21) water molecules with a diameter of $60 \AA$ $(10,969$ total atoms). All surface water molecules were subjected to deformable boundary forces (22) [approximated by a quartic polynomial (18)] and coupled to a heat bath of $300 \mathrm{~K}(17)$ with a coupling coefficient $(\beta)$ of $52 \mathrm{ps}^{-1}$. Additionally, all atoms were weakly coupled to the heat bath $\left(\beta=1 \mathrm{ps}^{-1}\right)$. After minimization, the system was equilibrated for 500 ps because it exhibited relaxations for more than 300 ps. After relaxation, the protein backbone atoms showed a root mean square deviation from the $x$-ray structure of $2.6 \AA$. Closer inspection revealed that a large frac- tion of the observed deviation arose from two loops (residues 65 to 70 and 112 to 122) at the tetramer interface, both of which are flexible in our monomer model but fixed by intermolecular interactions in the tetramer. The geometry of the binding pocket in our model does not deviate significantly from that of the $x$-ray structure (root mean square deviation, $1.5 \AA$ ). Structures randomly extracted from a subsequent MD run of 20 ps in duration were used for the simulations described in the text.

11. This choice allows thermal fluctuations for $z_{\mathrm{O} 2}$ of roughly $0.4 \AA$, a value commonly observed for unperturbed atomic fluctuations in proteins. Therefore, the thermal motion of the pulled atom is essentially unaffected by the spring potential.

12. We note that thermal fluctuations entail a logarithmic dependency of the rupture force on the pulling velocity (2); however, for the large velocities studied in our simulations, a linear dependency due to friction dominates. This friction heats the binding region by, at most, $3 \mathrm{~K}$ in the case of our slowest simulation. That heat is then effectively transferred to the heat bath; see (10).

13. H. Frauenfelder, G. A. Petsko, D. Tsernoglou, Nature 280, 558 (1979).

14. This simulation covered $1 \mathrm{~ns}$, within which the cantilever was moved by $15 \AA$. Considering the structural heterogeneity mentioned above, one cannot expect the finest details of the simulation or of the force profile to be reproducible. Therefore, we restrict our discussion to those typical features, which appeared in several of our simulations. We emphasize, however, that many more interactions contribute to the binding forces than those discussed here and that the rupture process is more complex than our simplifying pictures may suggest.

15. All hydrogen bonds found after equilibration are also found in the $x$-ray structure [P. C. Weber et al., J. Am. Chem. Soc. 114, 3197 (1992)].

16. For subtle reasons, simple integration of our force profile does not yield correct equilibrium binding free energies. The question of whether and how these can be obtained from our simulations is, therefore, left for future discussion.

17. M. Eichinger, H. Grubmüller, H. Heller, User Manual for EGO VIII, Release 2.0 (Theoretische Biophysik, Institut für Medizinische Optik, Universität München, 1995). Available from Internet at URL (http://www.imo physik.uni-muenchen.de/ego.html $\rangle$.

18. B. R. Brooks et al., J. Comp. Chem. 4, 187 (1983).

19. A. T. Brünger, X-PLOR (Howard Hughes Medical Institute and Department of Molecular Biophysics and Biochemistry, Yale University, New Haven, CT, 1988).

20. A. Kitao, F. Hirata, N. Gō, J. Phys. Chem. 97, 10223 (1993).

21. W. L. Jorgenson, J. Chandrasekhar, J. D. Madura, J Chem. Phys. 79, 927 (1983).

22. C. L. Brooks III and M. Karplus, ibid., p. 6312.

23. The figure was prepared with the use of MOLSCRIPT [P. J. Kraulis, J. Appl. Crystallogr. 24, 946 (1991)]

24. The computed force profiles exhibit fast fluctuations caused by (artificial) resonance of our spring. Accordingly, by simply taking the maximum force from these unprocessed profiles, one overestimates the rupture force. Therefore, before computing rupture forces, we removed the fast fluctuations by smoothing the force profiles with a Gaussian distribution of 4 ps width; that width has been estimated from the decay of the position autocorrelation function of atom $\mathrm{O} 2$ (compare Fig. 1B). This approach introduces some arbitrariness into the force computation because the maximum force depends on the smoothing width. To quantify that uncertainty, we determined error bars by considering force profiles smoothed on the scale of 2 ps (providing an upper bound) and 8 ps (lower bound), respectively.

25. We thank H. Gaub for stimulating this work, V. Moy for explaining details of the AFM experiment, and M. Eichinger and $\mathrm{H}$. Heller for discussions and help with the program EGO. Supported by the Deutsche Forschungsgemeinschaft, grant SFB 143/C1. For more in formation see <http://www.imo.physik.uni-muenchen. $\mathrm{de} / \sim$ grubi/streptavidin.html $>$

1 November 1995; accepted 4 January 1996 